\title{
Nondestructive Evaluation of Plates Using Eddy Current Methods
}

\author{
David C. Dobson* \\ Department of Mathematics \\ Texas A\&M University \\ College Station, TX 77843-3368
}

\author{
Fadil Santosa ${ }^{\dagger}$ \\ School of Mathematics \\ University of Minnesota \\ Minneapolis, MN 55455
}

September 1996

\begin{abstract}
The problem of determining defects in structures using eddy current methods is investigated. The goal of this work is to assess the extent to which accurate quantitative information about damage can be extracted from the data. For simplicity, we consider the problem of inspecting a plate in two dimensions. Damage is modeled as a change in the plate's conductivity. Electromagnetic field is generated by a pair of wires, which also serves as a measurement probe. The resulting mathematical model is analyzed by first deriving an integral identity, and second, by linearizing the relation between the conductivity anomaly and the data. We show that the problem of reconstructing a conductivity anomaly from eddy current data is quite unstable, and a method based on leastsquares principle would fail to detect conductivity anomalies in some situation. An alternate method, one that obtains gross properties of the anomaly (in this case an average) is proposed, and is shown to be quite robust. This work is contains several numerical experiments which illustrate our main ideas.
\end{abstract}

\section{Introduction}

The eddy current method is a nondestructive evaluation technique to detect flaws in metalic specimens. It has found prevalent use in aircraft safety inspection procedure [11], as well as in inspection of pipings and vessels [8]. It works on the principles of electromagnetics. An electromagnetic field is generated by a probe that usually consists of a wire loop with current passing through it. The electromagnetic field is measured at the probe while it scans over the surface of a specimen. The idea is that the measured field would change as the probe passes over a flaw, and the change can be used to detect the presence of a flaw.

\footnotetext{
${ }^{*}$ Research partially supported by Air Force Office of Scientific Research, grant number F49620-95-1-0497.

${ }^{\dagger}$ Research partially supported by the National Science Foundation under grant DMS-9503114, Department of Energy under grant DE-FG02-94ER25225, the Airforce Office of Scientific Research URI-RIP grant F49620-93-1-0500 and grant F49620-95-1-0305.
} 
While this nondestructive evaluation method has been quite effective in detecting the presence of flaws, it is generally not used for obtaining quantitative information about the flaws. In order to do so, we need to take two steps: (1) model the flaw quantitatively, for example, as a change in conductivity in the metal, and (2) understand the relationship between the parameters describing damage and eddy current data.

The present work is an attempt to characterize how reliably quantitative information about the flaw can be extracted from eddy current data. The ultimate goal is to develop a computational method by which eddy current data can be processed to provide quantitative damage information. Such a method would add value to the existing technology.

There has been some attempt to perform quantitative nondestructive evaluation using eddy current methods. The methods can be roughly separated into two classes: (i) damage modeled by material property change, (ii) damage modeled as cracks or voids. A leastsquares data fitting approach for determining changes in conductivity has been proposed by Sabbagh et al [10]. Auld et al [3] developed a method to determine the dimensions of a surface breaking semi-elliptical crack from eddy current data. In a recent work, Luong and Santosa, studying the nondestructive problem in corrosion inspection, developed a method for determining material loss profile in a plate [9].

In this work, we begin with a conductivity anomaly model for the flaw and develop a mathematical description for the relationship between the flaw and the data. An integral identity, which provides a framework for understanding the problem, is derived. The integral identity also provides a way of obtaining a linearized relation between the conductivity anomaly and the data. By concentrating on the simple two-dimensional plate geometry, we are able to obtain an explicit expression for a weighting function which lies at the heart of the linearized problem. This allows us to study the behavior of the problem in detail in several numerical computations.

Our findings are that the problem of determining conductivity anomalies from eddy current data is quite unstable. We propose an approach where we attempt to extract gross features information about the unknown, in this case an average. We are able to show that such information can be obtained reliably from the data. The method consists first in determining a convolution kernel. Once the kernel is found, we can (1) assess the ability of the method to extract information, and (2) convolve the data with the kernel to produce the desired information. The method extends in a straightforward manner to three dimensional plates. Indeed, because of the convolutional nature of the approach, very fast algorithms for three-dimensional data processing is prossible.

\section{Model problem}

We will consider a two dimensional problem for simplicity. The plate specimen being inspected is an infinite plate of thickness $h$ as depicted in Figure 1. We assume the flaw is localized. The eddy current probe is a pair of wires parallel to the $z$-axis, hovering at a distance $t$ above the plate at horizontal position $y_{0}$. The wires are separated by a distance of $2 d$ as indicated. Alternating current, at frequency $\omega$ and amplitude $I$, runs in opposite directions in the two wires. 


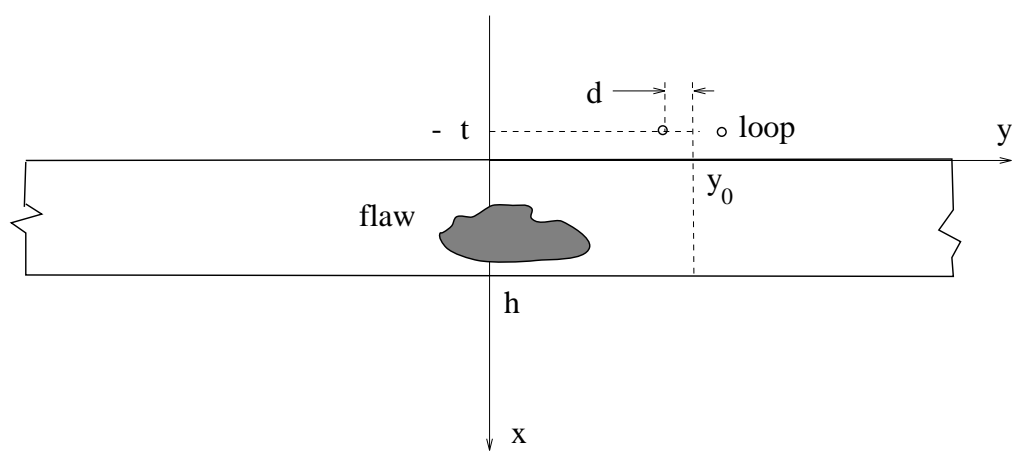

Figure 1: Idealized setup two dimensional setup. Loop impedance is collected as the probe scans over the specimen. The problem is to obtain quantitative information about the flaw from the data.

As the probe is moved across the surface, the loop impedance changes because of the presence of the flaw. The eddy current data, from which we would like to infer quantitative information about the flaw, is the impedance in in the loop as a function of $y_{0}$. Often, one would use several frequencies to probe and measure corresponding impedance at several discrete positions $y_{0 j}$, hence the data are

$$
Z\left(y_{0 j} ; \omega_{k}\right)=g_{j k} \text { for } j=1, \cdots, m, k=1, \cdots, n,
$$

where $Z\left(y_{0 j} ; \omega_{k}\right)$ is the loop impedance at loop location $y_{0 j}$ and probe frequency $\omega_{k}$.

Let $\boldsymbol{E}$ and $\boldsymbol{H}$ be the electric and magnetic fields. Because of the two-dimensional assumption, both fields depend on coordinates $(x, y)$. Moreover, current sources due to the two wires of the probe imply that

$$
\boldsymbol{E}=\left(0,0, E_{z}\right) \text { and } \boldsymbol{H}=\left(H_{x}, H_{y}, 0\right) .
$$

We assume (for simplicity only) that the permeability and permittivity are constant in both the air and the metal plate, and denote them by $\epsilon$ and $\mu$ respectively.

The presence of the flaw is modeled by a change in the conductivity. This may be justified in situations where damage is caused by corrosion, which has altered the material. A phenomenological relation between conductivity and extent of damage needs to be established. This is beyond the scope of this work. We assume here that there is a direct correlation between conductivity change and damage.

The conductivity $\sigma$ takes the form

$$
\sigma=\left\{\begin{array}{ll}
0 & \text { for } \quad(x, y) \in \text { air } \\
\sigma_{0}+\delta \sigma(x, y) & \text { for } \quad(x, y) \in \text { plate }
\end{array} .\right.
$$

The conductivity of the metal plate $\sigma_{0}$ is known. The perturbation $\delta \sigma$ is meant to represent the change in conductivity caused by the presence of the flaw. We assume that $\delta \sigma$ has compact support. 
Maxwell's equations govern the interaction of the probe with the surrounding medium, and are given by

$$
\begin{aligned}
\nabla \times \boldsymbol{E} & =i \omega \mu \boldsymbol{H} \\
\nabla \times \boldsymbol{H} & =(-i \omega \epsilon+\sigma) \boldsymbol{E}+\boldsymbol{J}
\end{aligned}
$$

Here, $\omega$ is the probing frequency. The current source $\boldsymbol{J}$ which models the probe is given by

$$
J_{x}=J_{y}=0, \quad J_{z}=I \delta(x+t)\left[\delta\left(y-y_{0}+d\right)-\delta\left(y-y_{0}-d\right)\right] .
$$

Keep in mind that in collecting data, $y_{0}$ will be changed.

The data, which is the impedance in the loop, is given by Faraday's law [7]

$$
Z\left(y_{0} ; \omega\right)=\frac{i \omega \mu}{I} \int_{y_{0}-d}^{y_{0}+d} H_{x}(-t, y) d y
$$

Given a conductivity perturbation $\delta \sigma$, we can find the loop impedance by solving (3) and computing (5). The inverse problem for this nondestructive evaluation method is to find $\delta \sigma$ from the data (1) and the model (3-5). In practice, the data for the problem is the difference between the impedance reading $Z\left(y_{0} ; \omega\right)$ of the inspected specimen and that of a flawless specimen. In our problem, the impedance in the flawless specimen, which is a uniform plate, is independent of probe position $y_{0}$. The value can be obtained from measurement.

\section{$3 \quad$ An integral identity}

In this section, we derive an integral identity that relates the impedance difference to the conductivity changes. This relation forms the basis for our analysis and computational method. There is a similar identity which is often used in electrical impedance tomography, and goes by the name of Calderon identity [5].

To set up this calculation, we need to work with the electromagnetic fields when the specimen is flawless. We call them background fields and denote them by $\left(\boldsymbol{E}^{b}, \boldsymbol{H}^{b}\right)$. They satisfy Maxwell's equation, given by

$$
\begin{aligned}
\nabla \times \boldsymbol{E}^{b} & =i \omega \mu \boldsymbol{H}^{b} \\
\nabla \times \boldsymbol{H}^{b} & =\left(-i \omega \epsilon+\sigma^{b}\right) \boldsymbol{E}^{b}+\boldsymbol{J} .
\end{aligned}
$$

The conductivity is given by

$$
\sigma^{b}=\left\{\begin{array}{ll}
\sigma_{0} & \text { for } \quad 0 \leq x \leq h,-\infty<y<\infty \\
0 & \text { for } \quad x<0,-\infty<y<\infty \\
0 & \text { for } \quad x>h,-\infty<y<\infty
\end{array} .\right.
$$

The current $\boldsymbol{J}$ has been specified in (4). The fields when the flaw is present can be written as

$$
\boldsymbol{E}=\boldsymbol{E}^{b}+\boldsymbol{E}^{s} \text { and } \boldsymbol{H}=\boldsymbol{H}^{b}+\boldsymbol{H}^{s} .
$$


We use superscript $s$ for scattered fields to be consistent with the scattering literature. From (6) it follows that $\left(\boldsymbol{E}^{s}, \boldsymbol{H}^{s}\right)$ satisfy

$$
\begin{aligned}
\nabla \times \boldsymbol{E}^{s} & =i \omega \mu \boldsymbol{H}^{s} \\
\nabla \times \boldsymbol{H}^{s} & =\left(-i \omega \epsilon+\sigma^{b}\right) \boldsymbol{E}^{s}+\delta \sigma \boldsymbol{E} .
\end{aligned}
$$

Note that the variable multiplying $\delta \sigma$ is the electric field when the flaw is present. Since we are in a two-dimensional setting, appropriate radiation conditions for $\left(\boldsymbol{E}^{s}, \boldsymbol{H}^{s}\right)$ are

$$
\begin{array}{ll}
\left(\nu \times \boldsymbol{E}^{s}\right)-\sqrt{\frac{\mu}{\epsilon}} \boldsymbol{H}^{s}=o\left(\frac{1}{\sqrt{R}}\right), \quad \boldsymbol{E}^{s}=\mathcal{O}(1 / \sqrt{R}), \\
\left(\nu \times \boldsymbol{H}^{s}\right)+\sqrt{\frac{\epsilon}{\mu}} \boldsymbol{E}^{s}=o\left(\frac{1}{\sqrt{R}}\right), \quad \boldsymbol{H}^{s}=\mathcal{O}(1 / \sqrt{R}),
\end{array}
$$

where in the half-space $\{x<0\}, \nu$ is the unit outward normal vector to a ball of radius $R$ centered at the origin. In the half-space $\{x>h\}$, the same condition applies with the ball centered at $(h, 0)$. In the conductive region $\{0 \leq x \leq h\}$, we specify that the fields must decay as $|y| \rightarrow \infty$.

We introduce a pair of trial fields $(\boldsymbol{F}, \boldsymbol{G})$ satisfying

$$
\begin{aligned}
\nabla \times \boldsymbol{F} & =i \omega \mu \boldsymbol{G}+\boldsymbol{L} \\
\nabla \times \boldsymbol{G} & =\left(-i \omega \epsilon+\sigma^{b}\right) \boldsymbol{F}
\end{aligned}
$$

We will specify $\boldsymbol{L}$ later. As with the scattered field $\left(\boldsymbol{E}^{s}, \boldsymbol{H}^{s}\right)$, a radiation condition is necessary:

$$
\begin{array}{ll}
(\nu \times \boldsymbol{F})-\sqrt{\frac{\mu}{\epsilon}} \boldsymbol{G}=o\left(\frac{1}{\sqrt{R}}\right), \quad \boldsymbol{F}=\mathcal{O}(1 / \sqrt{R}), \\
(\nu \times \boldsymbol{G})+\sqrt{\frac{\epsilon}{\mu}} \boldsymbol{F}=o\left(\frac{1}{\sqrt{R}}\right), \quad \boldsymbol{G}=\mathcal{O}(1 / \sqrt{R}) .
\end{array}
$$

Next, we multiply the first equation in (7) by $\boldsymbol{G}$ and the second by $\boldsymbol{F}$, and multiply the first equation in (9) by $\boldsymbol{H}^{s}$ and the second by $\boldsymbol{E}^{s}$. The resulting four expressions are combined to yield

$$
\boldsymbol{G} \cdot \nabla \times \boldsymbol{E}^{s}+\boldsymbol{F} \cdot \nabla \times \boldsymbol{H}^{s}-\boldsymbol{H}^{s} \cdot \nabla \times \boldsymbol{F}-\boldsymbol{E}^{s} \cdot \nabla \times \boldsymbol{G}=\delta \sigma \boldsymbol{E} \cdot \boldsymbol{F}-\boldsymbol{L} \cdot \boldsymbol{H}^{s} .
$$

The identity simplifies to

$$
\nabla \cdot\left(\boldsymbol{E}^{s} \times \boldsymbol{G}\right)+\nabla \cdot\left(\boldsymbol{H}^{s} \times \boldsymbol{F}\right)=\delta \sigma \boldsymbol{E} \cdot \boldsymbol{F}-\boldsymbol{L} \cdot \boldsymbol{H}^{s} .
$$

Let $B(x, R)$ denote the ball in $\mathbb{R}^{2}$ centered at $(x, 0)$. We define the "elongated ball" $B_{R}=B(0, R) \cup\{(x, y): 0 \leq x \leq h, \quad|y| \leq R\} \cup B(h, R)$. The expression above is now integrated over $B_{R}$. Using the divergence theorem, we get

$$
\int_{\partial B_{R}} \nu \cdot\left(\boldsymbol{E}^{s} \times \boldsymbol{G}\right)+\nu \cdot\left(\boldsymbol{H}^{s} \times \boldsymbol{F}\right) d s(x, y)=\int_{B_{R}} \delta \sigma \boldsymbol{E} \cdot \boldsymbol{F}-\boldsymbol{L} \cdot \boldsymbol{H}^{s} d x d y .
$$


From the radiation conditions (8a) and (10a) it follows that

$$
\left.\nu \cdot\left(\boldsymbol{E}^{s} \times \boldsymbol{G}+\boldsymbol{H}^{s} \times \boldsymbol{F}\right)\right|_{\partial B_{R}}=o(1 / R) .
$$

Now, letting $R \rightarrow \infty$ and rearranging, we arrive at

$$
\int_{\mathbb{R}^{2}} \boldsymbol{L} \cdot \boldsymbol{H}^{s} d x d y=\int_{-\infty}^{\infty} \int_{0}^{h} \delta \sigma \boldsymbol{E} \cdot \boldsymbol{F} d x d y
$$

The identity in (11) is made useful by choosing $\boldsymbol{L}$ to produce, on the lefthand side, the loop impedance. To this end, we select $\boldsymbol{L}=\left(L_{x}, 0,0\right)$ with

$$
L_{x}(x, y)=\delta(x+t)\left[\mathrm{H}\left(y-y_{0}+d\right)-\mathrm{H}\left(y-y_{0}-d\right)\right]
$$

where $\mathrm{H}(\cdot)$ is the Heaviside function. Under this choice, the lefthand side of (11) becomes

$$
\int_{y_{0}-d}^{y_{0}+d} H_{x}^{s}(-t, y) d y
$$

To compute the loop impedance in (5) we use the total magnetic field. Therefore, to compute changes in the loop impedance, we need to use the scattered field. Thus

$$
\delta Z\left(y_{0} ; \omega\right)=\frac{i \omega \mu}{I} \int_{y_{0}-d}^{y_{0}+d} H_{x}^{s}(-t, y) d y .
$$

and we make the identification that with the choice $\boldsymbol{L}$ in (12), equation (11) amounts to

$$
\delta Z\left(y_{0} ; \omega\right)=\frac{i \omega \mu}{I} \int_{0}^{h} \int_{-\infty}^{\infty} \delta \sigma \boldsymbol{E} \cdot \boldsymbol{F} d x d y .
$$

We will use (13) as a basic tool for our analysis and numerical computation. Note that the derivation carries over with a few modifications to the full three dimensional case.

\section{Linearized problem}

In this section, we formulate the linearized eddy current imaging problem in two dimensions. We exploit the simplicity of the two dimensional fields by considering the equations satisfied by the scalar field $E_{z}(x, y)$. From Maxwell's equations (6), we know that

$$
-\triangle E_{z}^{b}=\left(\omega^{2} \mu \epsilon+i \omega \mu \sigma^{b}\right) E_{z}^{b}+i \omega \mu J_{z} .
$$

Observe that from (9), we can show that

$$
-\triangle F_{z}=\left(\omega^{2} \mu \epsilon+i \omega \mu \sigma^{b}\right) F_{z}-\partial_{y} L_{x}
$$

Recall that $L_{x}$ has been chosen in (12) and upon calculating its partial with respect to $y$, we find

$$
\begin{aligned}
\partial_{y} L_{x} & =\delta(x+t)\left[\delta\left(y-y_{0}+d\right)-\delta\left(y-y_{0}-d\right)\right] \\
& =J_{z} / I
\end{aligned}
$$


Therefore, we can identify

$$
F_{z}=\frac{i E_{z}^{b}}{I \omega \mu}
$$

Inserting this result in (13), we find

$$
\delta Z\left(y_{0} ; \omega\right)=-\frac{1}{I^{2}} \int_{0}^{h} \int_{-\infty}^{\infty} \delta \sigma(x, y) E_{z} E_{z}^{b} d x d y .
$$

The formula in (15) is exact. To obtain a formal linearization, we replace $E_{z}$ by the background field $E_{z}^{b}$. Thus the linearized forward map is

$$
\delta Z\left(y_{0} ; \omega\right)=-\frac{1}{I^{2}} \int_{0}^{h} \int_{-\infty}^{\infty} \delta \sigma E_{z}^{b}\left(x, y ; y_{0}, \omega\right)^{2} d x d y
$$

The linearized inverse problem can be posed as a "finite moment problem":

Find the function $\delta \sigma(x, y)$ given its weighted averages $g_{j k}$ in the equation

$$
-\frac{1}{I^{2}} \int_{0}^{h} \int_{-\infty}^{\infty} \delta \sigma(x, y) E_{z}^{b}\left(x, y ; y_{0 j}, \omega_{k}\right)^{2} d x d y=g_{j k}, \quad \text { for } \quad j=1, \cdots, m, k=1, \cdots, n .
$$

We now proceed to give a formula for $E_{z}^{b}\left(x, y ; y_{0}, \omega\right)$. Because of the exponentially decaying solution for $x>0$, it is convenient to rescale the problem in terms of skin depths. Traditionally, skin depth in a conducting medium is defined as [4]

$$
d_{\text {skin }}=\sqrt{\frac{2}{\omega \mu \sigma_{0}}} .
$$

We find it convenient to use the scaling

$$
x=\frac{x^{\prime}}{\sqrt{\omega_{0} \mu \sigma_{0}}} \text { and } \omega=r \omega_{0},
$$

where $\omega_{0}$ is a reference frequency, and $\sigma_{0}$ is the conductivity of the metal. Under this scaling, we are measuring distances in numbers of skin depths at some reference frequency. The two parameters we need to carry are

$$
r=\frac{\omega}{\omega_{0}} \quad \text { and } \quad s=\frac{\epsilon \omega_{0}}{\sigma_{0}} .
$$

We define $t^{\prime}=t \sqrt{\omega_{0} \mu \sigma_{0}}, d^{\prime}=d \sqrt{\omega_{0} \mu \sigma_{0}}$, and $h^{\prime}=t \sqrt{\omega_{0} \mu \sigma_{0}}$.

We will construct $E_{z}$ by solving for $G\left(x^{\prime}, y^{\prime}\right)$ satisfying

$$
-\triangle^{\prime} G=k^{2} G+\delta\left(x^{\prime}+t^{\prime}\right)\left[\delta\left(y^{\prime}+d^{\prime}\right)-\delta\left(y^{\prime}-d^{\prime}\right)\right]
$$

where

$$
k^{2}= \begin{cases}r^{2} s & \text { for } x^{\prime}<0,-\infty<y^{\prime}<\infty \\ r^{2} s+i r & \text { for } 0 \leq x^{\prime} \leq h^{\prime},-\infty<y^{\prime}<\infty \\ r^{2} s & \text { for } x^{\prime}>h^{\prime},-\infty<y^{\prime}<\infty\end{cases}
$$


We need to impose continuity of $G$ and $\partial_{x^{\prime}} G$ across the interfaces at $x^{\prime}=0$ and $x^{\prime}=h^{\prime}$.

The solution for $G$ is obtained by first taking the Fourier transform of the equation (19) in $y$. The solution for the transformed variable, $\hat{G}\left(x^{\prime}, \eta\right)$ is easily found. Of particular interest is the field for $0 \leq x^{\prime} \leq h^{\prime}$, which, after some lengthy calculations, is given by

$\hat{G}\left(x^{\prime}, \eta\right)=\frac{2 e^{i \beta_{0} t} \sin \eta d^{\prime}}{e^{-i \beta_{1} h^{\prime}}\left(\beta_{0}+\beta_{1}\right)^{2}-e^{i \beta_{1} h^{\prime}}\left(\beta_{0}-\beta_{1}\right)^{2}}\left\{\left(\beta_{0}-\beta_{1}\right) e^{-i \beta_{1}\left(x^{\prime}-h^{\prime}\right)}-\left(\beta_{0}+\beta_{1}\right) e^{i \beta_{1}\left(x^{\prime}-h^{\prime}\right)}\right\}$,

where

$$
\beta_{0}^{2}=r^{2} s-\eta^{2}, \quad \beta_{1}^{2}=\left(r^{2} s+i r\right)-\eta^{2} .
$$

The field $G\left(x^{\prime}, y^{\prime}\right)$ is the inverse Fourier transform of $\hat{G}\left(x^{\prime}, \eta\right)$.

The desired background field, in the scaled coordinates, is given by

$$
E_{z}^{b}\left(x^{\prime}, y^{\prime} ; y_{0}^{\prime}, r\right)=\frac{i r I}{\sigma_{0}} G\left(x^{\prime}, y^{\prime}-y_{0}^{\prime} ; r\right) .
$$

We have explicitly displayed the dependence of $G$ on $r$ (hence, on $\omega$ ). Moreover, we will view $E^{b}$ as dependent on the parameter $r$ as well. Hence forth, we will drop the primes on our independent variables and work with the scaled problem.

Another approach to finding the linearized relation between the impedance changes and the conductivity perturbation has been proposed in [1,2]. Briefly, the approach is to view the solution for $(\boldsymbol{E}, \boldsymbol{H})$ of (3) in the form $\boldsymbol{E}=\boldsymbol{E}^{b}+\boldsymbol{E}^{s}$ and $\boldsymbol{H}=\boldsymbol{H}^{b}+\boldsymbol{H}^{s}$ as a two-term perturbation series solution. By assuming that $\delta \sigma$ and $\left(\boldsymbol{E}^{s}, \boldsymbol{H}^{s}\right)$ are small, one can obtain a partial differential equation relating the perturbational field with the background field and the conductivity perturbation. One can then solve for $\left(\boldsymbol{E}^{s}, \boldsymbol{H}^{s}\right)$ explicitly and evaluate the integral of $H_{x}^{s}$ to obtain the desired change in the loop impedance.

We prefer our approach because it is quite general and easier to compute with. Furthermore, our method sheds some valuable insight into how the changes in the loop impedance is produced by the conductivity perturbation, and how the inverse problem is illposed.

\section{$5 \quad$ Stability and resolution}

It is instructive to visualize the electrical field caused by a probe over a uniform (undamaged) plate in order to understand the source of the illposedness of the inverse problem. Consider the situation where the normalized thickness is $h=1$, which means that the skin depth is $\sqrt{2}$ times the plate thickness. Let us choose a probe consisting of a pair of wires hovering at $t=0.2$ above the plate and separated by half distance $d=0.2$. In Figure 2, we display the contours of the imaginary part of the generated electrical field $E_{z}^{b}$ at frequency ratios $r=1$ and $r=6$, and $s=0.01$. Note that higher $r$ values correspond to higher frequencies, therefore, it is not surprising that for $r=6$, the electrical field decays more rapidly into the plate that the field at $r=1$.

Next, note that in the the linearized inverse problem, we will be given integrals of the desired conductivity perturbations against weights $E_{z}^{b}\left(x, y ; y_{0 j}, \omega_{k}\right)^{2}$, so it is also instructive to visualize the weight functions. The integrations are over the extent of the plate. Observe 

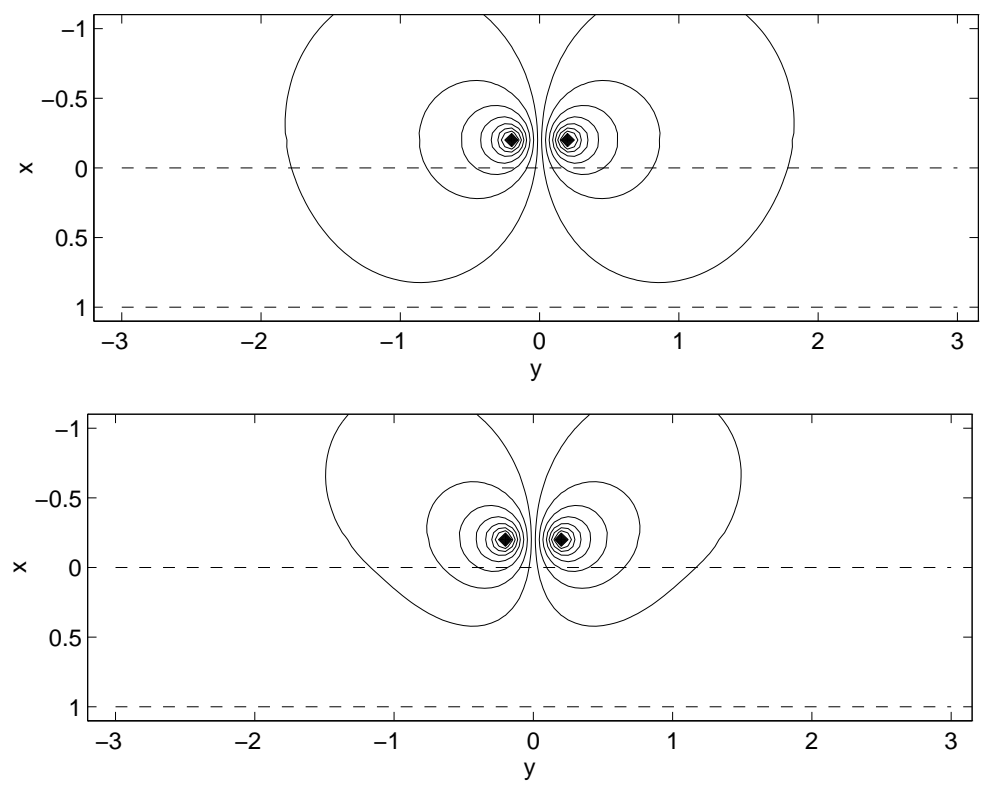

Figure 2: Contours of the imaginary part of the electrical field for $r=1$ and for $r=6$, with $s=0.01$. The plate surfaces are shown as horizontal dashed lines. Higher $r$ values correspond to high frequencies.

that the weight functions are simply translated when $y_{0}$ is varied. We show grey level plots of the real part of $E_{z}^{b}\left(x, y ; 0, \omega_{k}\right)^{2}$ for $r=1$ and $r=6$ in Figures $3 \mathrm{a}$ and $3 \mathrm{~b}$. Note that the imaginary parts are smaller in magnitude. The weight functions decay very rapidly away from the top of the plate, with the decay rate increasing as frequency (in this case $r$ ) is increased.

The ramifications of the nature of these weight functions are clear: because the weight functions are nearly null near the bottom of the plate, conductivity perturbations near the bottom of the plate are going to be very difficult to reconstruct accurately and stably. An assessment of the instability can be made through the singular value decomposition.

To this end, consider a data set consisting of measurements of loop impedances over the top of the plate spanning the line segment $[-2,2]$ at intervals of 0.2 . That is we have loop impedance readings at coordinate $\left(-0.2, y_{0 j}\right), y_{0 j}=-2+0.2(j-1), j=1, \cdots, 21$. At these measurement points, loop impedance is recorded for 6 frequencies corresponding to $r=1,2, \cdots, 6$. The forward map takes conductivity perturbations over the domain $[0,1] \times[-12.8,12.7]$ to the loop impedances at the measurement points. The conductivity perturbations are assumed to be piecewise constant over regular pixels of size 0.1-by-0.1. We further discretize the weight functions as piecewise constants over the same grid. This renders the integrations into summations. The resulting complex linear system of equations are treated as a pair of linear systems for the real and imaginary parts. This was done in order to maintain the real nature of the conductivity perturbations $\delta \sigma$.

It can be seen in Figure 4 that the singular values of the discrete linear operator decay 

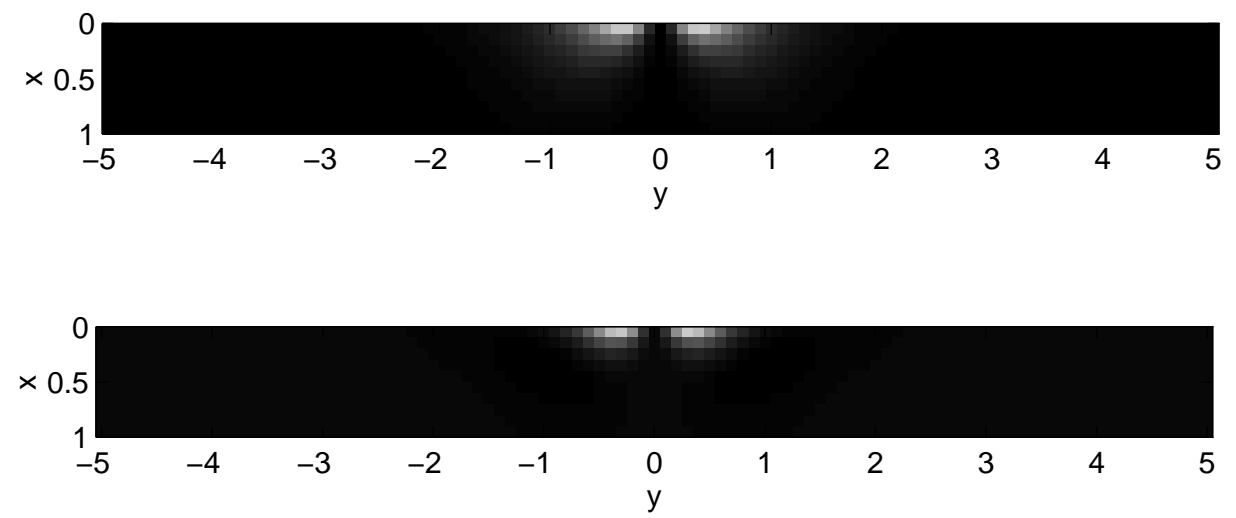

Figure 3: Grey scale plots of the real part of the weight functions for $r=1$ and for $r=6$, with $s=0.01$. Higher $r$ values correspond to high frequencies.

at a nearly exponential rate. Therefore, it is inevitable that in order to have stability in the inversion, we must sacrifice resolution.

To understand the limitations caused by the inherent instability, consider the problem of reconstructing a conductivity perturbation distribution $\delta \sigma$. Let us consider solving the linear system in (17) given the righthand side corresponding to some function $\delta \sigma(x, y)$. We can assume that the data are consistent with some conductivity perturbation. Given the data, we apply the pseudo-inverse computed using the singular value decomposition (see e.g., [6]. We control the stability by specifying the condition number in the pseudo-inverse.

We show the reconstructions of a 'point defect' under various condition numbers. To give a sense of the forward map, we display the data at the top of Figure 5. Shown are the real parts of the loop impedances at the different measurement locations at the 6 frequencies. Below the figure for the data, we display the point defect conductivity perturbations in question for reference. The reconstructions are displayed next in the figure. In viewing these reconstruction, refer to the true conductivity perturbation shown for comparison. The condition numbers for the reconstructions are 100, 1000, and 10000. Larger condition numbers give better reconstruction at the cost of greater instability. Further experiments show that for a given condition number, reconstructions of point defects degrade as the defect location is moved away from the top surface. This is expected because of the nature of the weight function which die off exponentially away from the top surface.

The results give us a false sense of security that resolution loss is controllable by playing it against stability. The next example shows that the situation is much worse than the previous experiment demonstrates. In this experiment, we have 2 point defects - one near the top surface, and the other, near the bottom. The reconstructions at condition numbers 100, 1000, 10000 are shown in Figure 8. Note that the defect near the bottom is nearly invisible in the reconstructions. This is because the lower defect generates very small changes in data as shown in Figure 6a. Indeed the data in Figure 6a are nearly indistinguishable 


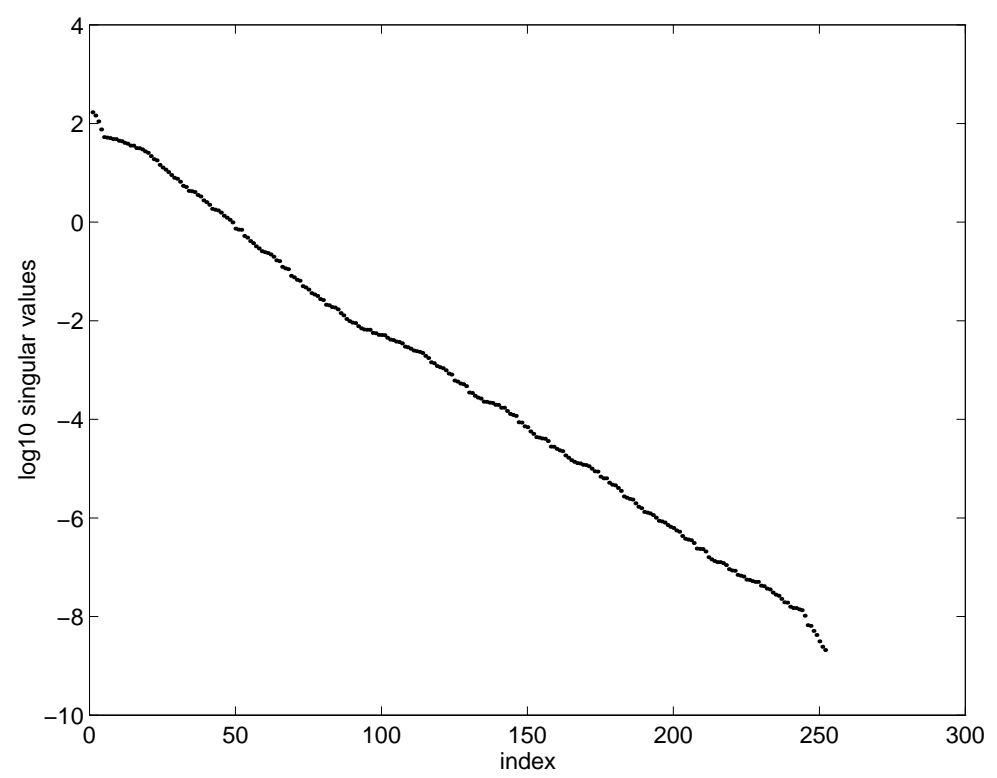

Figure 4: Singular value distribution of the discretized forward map. The map takes conductivities in the region $[0,1] \times[-12.8,12.7]$ to loop impedances at coordinates $\left(-0.2, y_{0 j}\right)$, $y_{0 j}=-2+0.2(j-1), j=1, \cdots, 21$. Note the near exponential decay rate of the singular values.

from those when the lower defect is absent. Moreover, the singular vectors corresponding to the smaller singular values are for conductivity perturbations near the bottom of the plate. Therefore, in computing the pseudo-inverse solution, the information about the conductivity perturbations near the bottom is lost altogether.

One can also estimate the loss of information with depth directly from the Fourier transformed Green's function (20) as follows. From the moment problem

$$
\int_{0}^{h} \int_{-\infty}^{\infty} \delta \sigma(x, y) E_{z}^{b}\left(x, y ; y_{0 j}, \omega_{k}^{2}\right)^{2} d x d y=g_{j k},
$$

consider a conductivity perturbation of the form $\delta \sigma(x, y)=\delta\left(x-x_{0}\right) e^{-i \lambda y}$. The response of the data to such a perturbation indicates how resolution in the Fourier domain depends on depth $x_{0}$. The response in terms of the scaled variables is

$$
\int_{-\infty}^{\infty} e^{-i \lambda y} E_{z}^{b}\left(x_{0}, y ; y_{0 j}, \omega_{k}^{2}\right)^{2} d y=\mathcal{F}\left(G\left(x_{0}, \cdot ; r\right)^{2}\right)(\lambda),
$$

where $\mathcal{F}$ represents the Fourier transform. We can then estimate

$$
\left|\mathcal{F}\left(G\left(x_{0}, \cdot ; r\right)^{2}\right)(\lambda)\right| \leq \int\left|\hat{G}\left(x_{0}, \lambda-\eta\right)\right|\left|\hat{G}\left(x_{0}, \eta\right)\right| d \eta,
$$

where $\hat{G}$ is given by (20). For $x_{0}>0$ and $|\eta|$ sufficiently large, one can bound $\left|\hat{G}\left(x_{0}, \eta\right)\right| \leq$ $C e^{-|\eta| x_{0}}$ where $C$ is independent of $x_{0}$ and $\eta$. It follows by direct calculation that the data 

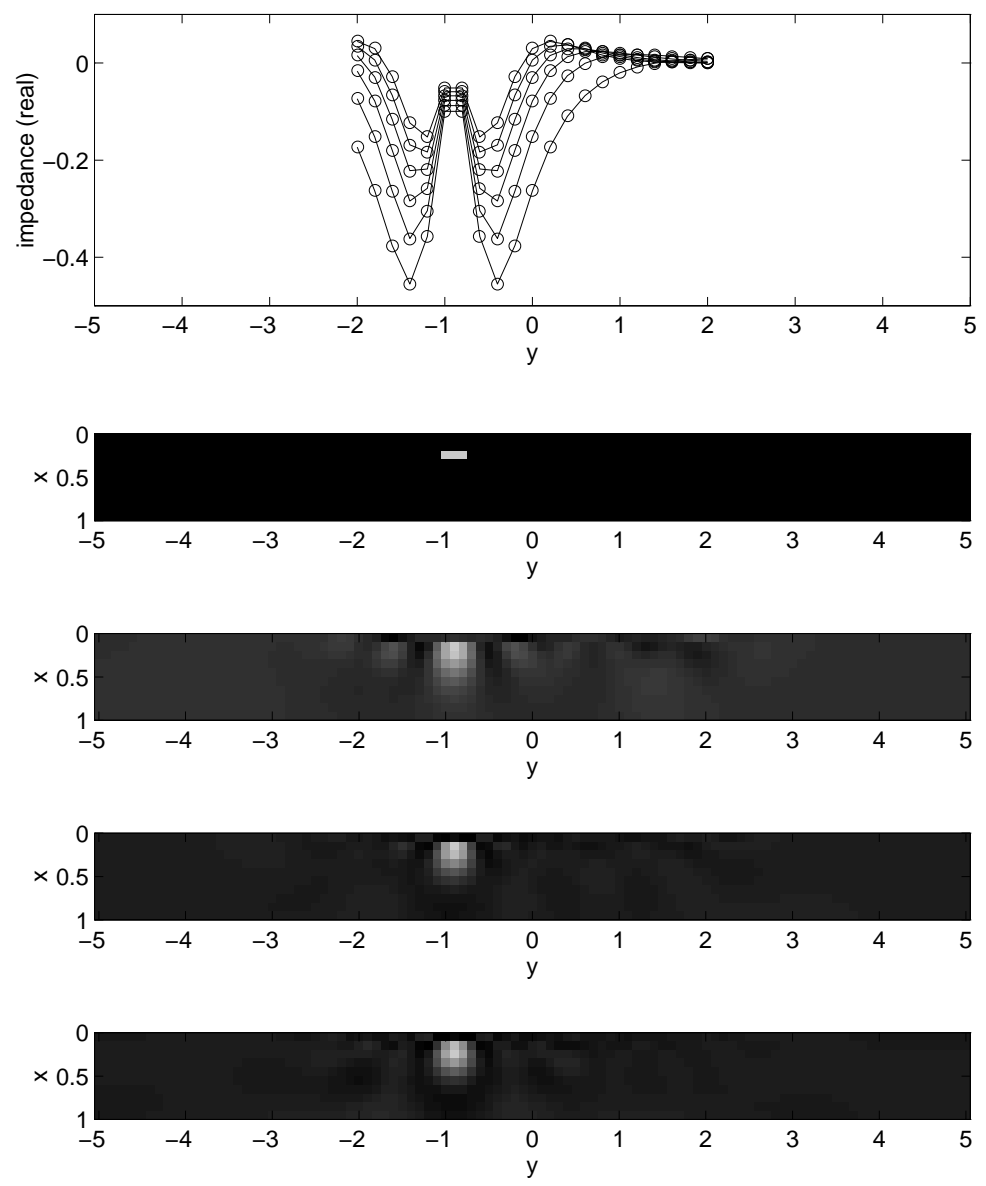

Figure 5: Reconstruction of a point defect under various condition numbers. The data corresponding to the conductivity perturbation is displayed at the top. The true conductivity perturbation is shown next. This is followed by reconstructions at using the pseudo-inverse with condition numbers 100, 1000, and 10000. Note that for this problem, resolution can be controlled by playing it against stability. 

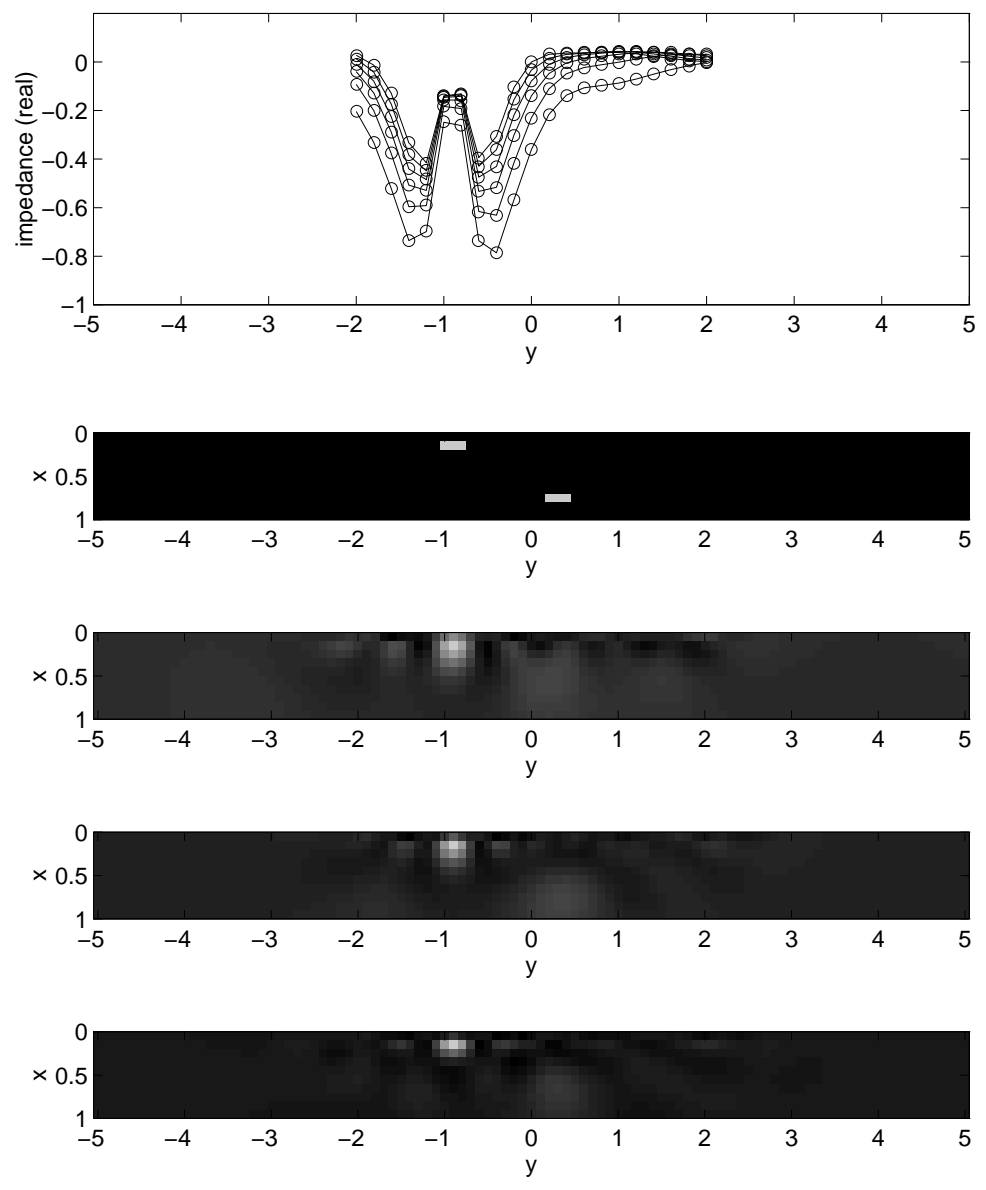

Figure 6: Reconstruction of 2 point defects under various condition numbers. The data corresponding to the conductivity perturbation is displayed at the top. Observe that the data contains little information from the lower anomaly. The true conductivity perturbation is shown next, followed by the reconstructions at condition numbers 100, 1000, and 10000 . Note that the lower point defect is lost in the reconstruction. 
response is bounded by

$$
C e^{-|\lambda| x_{0}}\left(1 / x_{0}+\lambda\right)
$$

Thus resolution in the Fourier domain decreases exponentially with depth, and for any positive depth, data response to oscillatory perturbations decays exponentially with frequency.

We conclude that any attempt to reconstruct the conductivity perturbation image may be unreliable because of the nature of the forward map, which only "sees" conductivity perturbations near the top. In the next section, we modify the problem and seek to find other attributes of the conductivity perturbations which can be stably obtained from the data.

\section{Obtaining vertical averages of conductivity}

Since obtaining conductivity perturbations from the data appears to be asking too much, we propose to modify our question and attempt to extract less information (but more stably) from the given data. Suppose that the desired conductivity perturbation is $\delta \sigma(x, y)$. We know we cannot reconstruct it robustly from the data. Let us attempt instead to find an approximate vertical average of the unknown

$$
\overline{\delta \sigma}(y)=\int_{-\infty}^{\infty} \int_{0}^{h} \delta \sigma(x, y) f\left(x, y-y^{\prime}\right) d x d y^{\prime} .
$$

Ideally, we would like the function $f\left(x, y-y^{\prime}\right)$ to be equal to

$$
\frac{1}{h} \delta\left(y^{\prime}-y\right),
$$

which would make $\overline{\delta \sigma(y)}$ the true vertical average of $\delta \sigma(x, y)$ at $y$. However, for reasons explained below, we can only attempt to make $f(x, y)$ resemble the desired delta function. For the moment, we simply require $\int_{-\infty}^{\infty} \int_{-h}^{h} f(x, y) d x d y=1$. Our construction will allow us to find $f(x, y)$ which is highly localized around $y=0$, and thus, yielding $\overline{\delta \sigma(y)}$ which is approximately equal to the vertical average at $y$.

Such information will be useful in nondestructive evaluation as it can locate the damage horiziontally (along the plate) and provide quantitative information about the damage at that location. It does not, however, provide information about the depth location of the damage.

Let us look at the linearized problem in (17). Suppose for the moment that we have continuous measurements. That is we wish to solve for $\delta \sigma(x, y)$ in

$$
\int_{0}^{h} \int_{-\infty}^{\infty} \delta \sigma(x, y) M_{k}\left(x, y-y_{0}\right) d x d y=g_{k}\left(y_{0}\right), \quad \text { for } \quad-\infty<y_{0}<\infty, k=1, \ldots, n,
$$

where

$$
M_{k}\left(x, y-y_{0}\right)=-\frac{1}{I^{2}} E_{z}^{b}\left(x, y ; y_{0}, \omega_{k}\right)^{2}
$$


This is justified due to the nature of the dependence of the electric field $E_{z}$ on $y$ and $y_{0}$. Consider the problem of finding a function $\Phi_{k}\left(y_{0}\right)$ such that

$$
\operatorname{Re}\left\{\sum_{k=1}^{n} \int_{-\infty}^{\infty} \Phi_{k}\left(y_{0}\right) M_{k}\left(x, y-y_{0}\right) d y_{0}\right\}=f(x, y) .
$$

The point is that if we can find such a function with the desired "delta-ness" property, then applying it to both sides of (22), summing, and integrating in $y_{0}$, will give us the approximate vertical average of the conductivity perturbation at $y=0$. Now, to find an approximate vertical average at $y=y^{\prime}$, we can apply $\Phi_{k}\left(y_{0}\right)$ to a translated version of $M_{k}\left(x, y-y_{0}\right)$, i.e.,

$$
\operatorname{Re}\left\{\sum_{k=1}^{n} \int_{-\infty}^{\infty} \Phi_{k}\left(y_{0}\right) M_{k}\left(x, y-y^{\prime}-y_{0}\right) d y_{0}\right\}=f\left(x, y-y^{\prime}\right) .
$$

In terms of the data, if $\Phi_{k}\left(y_{0}\right)$ is at our disposal, we have the inversion formula

$$
\overline{\delta \sigma}\left(y^{\prime}\right)=\operatorname{Re}\left\{\sum_{k=1}^{n} \int_{-\infty}^{\infty} \Phi_{k}\left(y_{0}\right) g_{k}\left(y^{\prime}+y_{0}\right) d y_{0}\right\} .
$$

This convolution takes the data and produces an approximate vertical average.

To find a solution to (23), we formally look for the least squares solution. That is, setting $\Phi=\left\{\Phi_{k}\right\}_{k=1}^{n}$, and defining the linear operator $\mathcal{M}$ by

$$
(\mathcal{M} \Phi)(x, y)=\operatorname{Re}\left\{\sum_{k=1}^{n} \int_{-\infty}^{\infty} \Phi_{k}\left(y_{0}\right) M_{k}\left(x, y-y_{0}\right) d y_{0},\right\}
$$

we consider the quadratic minimization

$$
\min _{\Phi} J(\Phi)=\int_{0}^{h} \int_{-\infty}^{\infty}|(\mathcal{M} \Phi)(x, y)-f(x, y)|^{2} d y d x
$$

The normal equation for $\Phi$ is

$$
\mathcal{M}^{*} \mathcal{M} \Phi=\mathcal{M}^{*} f
$$

or more explicitly,

$$
\begin{aligned}
\sum_{k=1}^{n} \int_{-\infty}^{\infty} \quad\left\{A_{k l}\left(y_{0}, y_{0}^{\prime}\right)\right. & \left.\operatorname{Re} \Phi_{k}\left(y_{0}\right)-B_{k l}\left(y_{0}, y_{0}^{\prime}\right) \operatorname{Im} \Phi_{k}\left(y_{0}\right)\right\} d y_{0} \\
& =\int_{-\infty}^{\infty} \int_{0}^{h} f(x, y) \operatorname{Re} M_{l}\left(x, y-y_{0}^{\prime}\right) d x d y \\
\sum_{k=1}^{n} \int_{-\infty}^{\infty} \quad\left\{C_{k l}\left(y_{0}, y_{0}^{\prime}\right)\right. & \left.\operatorname{Re} \Phi_{k}\left(y_{0}\right)-D_{k l}\left(y_{0}, y_{0}^{\prime}\right) \operatorname{Im} \Phi_{k}\left(y_{0}\right)\right\} d y_{0} \\
& =\int_{-\infty}^{\infty} \int_{0}^{h} f(x, y) \operatorname{Im} M_{l}\left(x, y-y_{0}^{\prime}\right) d x d y
\end{aligned}
$$



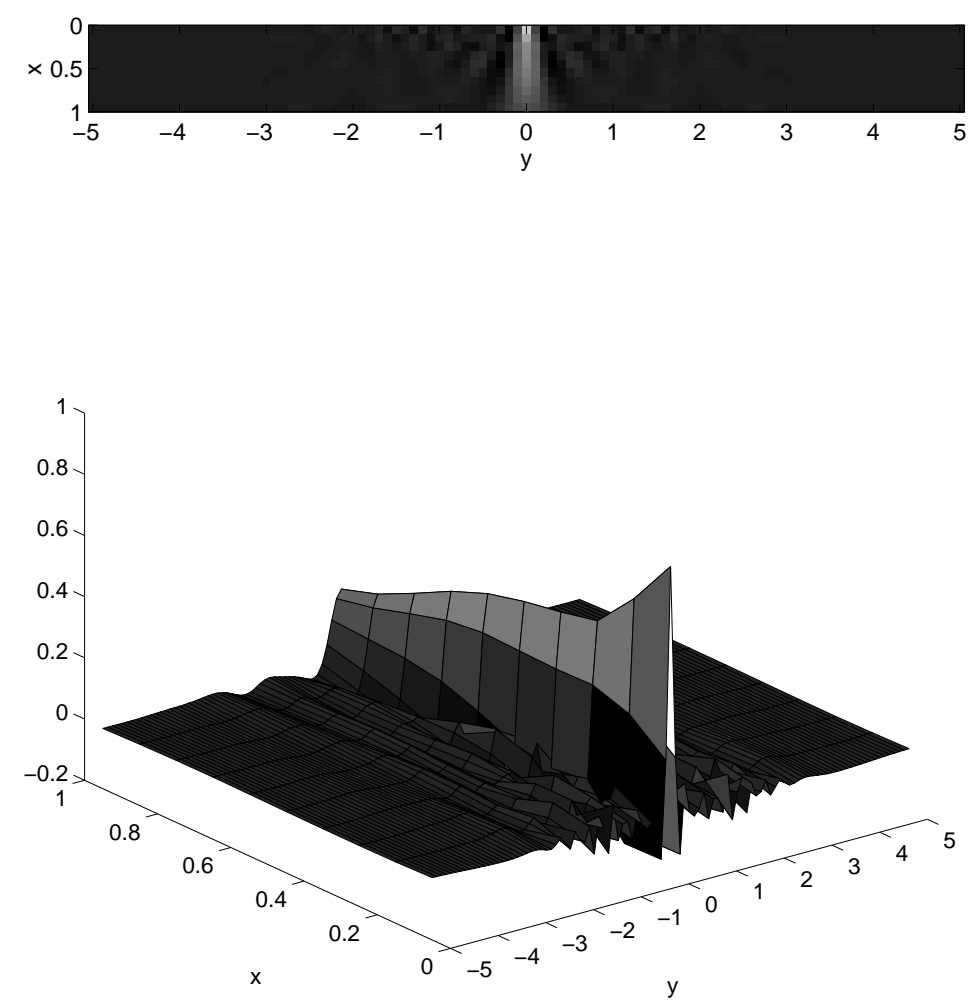

Figure 7: Approximate delta function from numerical experiment.

where

$$
\begin{aligned}
& A_{k l}\left(y_{0}, y_{0}^{\prime}\right)=\int_{-\infty}^{\infty} \int_{0}^{h} \operatorname{Re} M_{k}\left(x, y-y_{0}\right) \operatorname{Re} M_{l}\left(x, y-y_{0}^{\prime}\right) d x d y, \\
& B_{k l}\left(y_{0}, y_{0}^{\prime}\right)=\int_{-\infty}^{\infty} \int_{0}^{h} \operatorname{Im} M_{k}\left(x, y-y_{0}\right) \operatorname{Re} M_{l}\left(x, y-y_{0}^{\prime}\right) d x d y, \\
& C_{k l}\left(y_{0}, y_{0}^{\prime}\right)=\int_{-\infty}^{\infty} \int_{0}^{h} \operatorname{Re} M_{k}\left(x, y-y_{0}\right) \operatorname{Im} M_{l}\left(x, y-y_{0}^{\prime}\right) d x d y \\
& D_{k l}\left(y_{0}, y_{0}^{\prime}\right)=\int_{-\infty}^{\infty} \int_{0}^{h} \operatorname{Im} M_{k}\left(x, y-y_{0}\right) \operatorname{Im} M_{l}\left(x, y-y_{0}^{\prime}\right) d x d y .
\end{aligned}
$$

Note that for the derivation above to make sense, we must have $f \in L^{2}$, for otherwise (25) is not well defined. This technical restriction prevents the choice $f(x, y)=\delta(y) / h$, but does not prevent making $f$ an arbitrarily good approximation to $\delta$. Therefore, we formally replace $f(x, y)$ by $\delta(y) / h$ in $(26)$ and proceed with the calculation. A final assessment of how well we have done in approximating a true delta function can be made.

There are several practical issues to keep in mind in applying this method. First, notice that the weight function $M_{k}(x, y)$ decays rapidly in $y$ as shown in Figure 3 . This means 

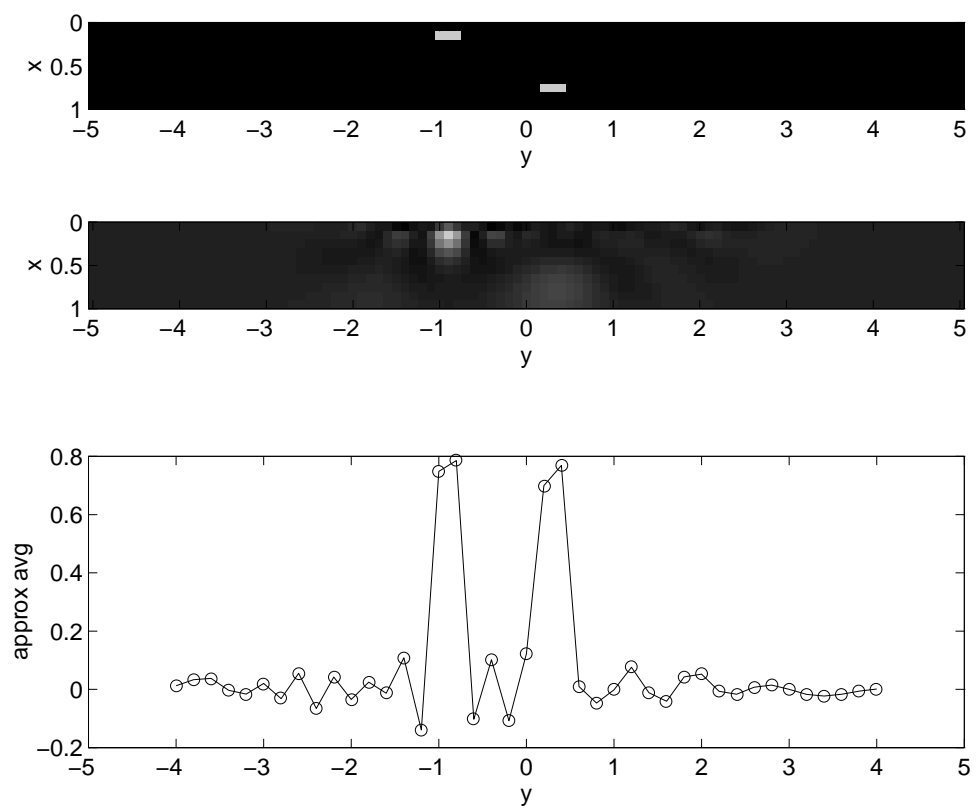

Figure 8: Shown at the top is the true conductivity perturbation. Second figure corresponds to a reconstruction at condition number 1000. Last plot is the approximate vertical average obtained by our method. Note that both defects were detected and estimated.

that we can truncate the integration in $y_{0}$ to a smaller interval. Once a $\Phi_{k}\left(y_{0}\right)$ function is found, one can check for its 'delta-ness' by considering the expression

$$
(\mathcal{M} \Phi)(x, y)=\operatorname{Re}\left\{\sum_{k=1}^{n} \int_{-\infty}^{\infty} \Phi_{k}\left(y_{0}\right) M_{k}\left(x, y-y_{0}\right) d y_{0} \cdot\right\}
$$

We can adjust the size of the interval in the integration for $y_{0}$ and the frequencies in order to find an acceptible approximation to the delta function $\delta(y)$. This is an important aspect of this method. It allows us to design the measurement procedure in order to obtain an acceptable result before the actual measurements take place.

As an example, we consider the same plate and probe geometry we have looked at up to this point. We assume that $y_{0}$ is given over an interval of size 4 , and at a sampling rate of 0.2 . We use 6 frequencies to probe the plate, corresponding to $r=1,2,3,4,5,6$. We then discretized (26) using piecewise constant approximation of $M_{k}(x, y)$ over regular pixels of size 0.1 -by-0.1. The integration in $y$ is truncated to $[-12.8,12.7]$. The resulting linear system is solved to obtain $\Phi_{k}\left(y_{0}\right)$. To check the result, we performed a 'delta-ness' verification as indicated previously. We obtained an approximate delta function displayed in Figure 7. It can be seen from the figure that the approximate delta function is very accurate near the top but is not as concentrated near the bottom. However, note that the main lobe decays slowly as a function of depth, and therefore, we expect this kernel to pick up approximate vertical averages accurately even if the conductivity anomany is located near the bottom of the plate. 
To assess the effectiveness of the approximate delta, we apply the computed function $\Phi_{k}\left(y_{0}\right)$ on a data set. The result is displayed in Figure 8. On the top, we show the true image of the point defects. The second plot shows an attempt to reconstruct the conductivity perturbation at condition number 1000 . Note that the bottom defect is lost in the reconstruction. The last plot shows the vertical conductivity average obtained with our method. It is clear that by asking less from the data, we are able to obtain a more reliable information.

\section{Discussion}

We have studied a two-dimensional version of an eddy current nondestructive evaluation of a plate. A flaw in the plate specimen is modeled as a conductivity anomaly. Our investigation shows that the conductivity anomaly cannot be reliably recovered from the eddy current

data. Indeed it appears possible for the method to miss a conductivity anomaly in some circumstances.

In order to combat the inherent illposedness of the method, we propose a "less-ismore approach" based on obtaining gross quantitative information about the flaw. In this study, we designed a method for finding an approximate vertical average of the conductivity anomaly directly from the data. The method has several desirable features, among them

- We can perform analysis to design the measurement procedure that would yield acceptable accuracy.

- The computational method is convolutional and can be implemented to produce fast algorithms.

- The method extends to three dimensions and to more complicated probes.

The preliminary results obtained in this work indicates that the present approach of obtaining gross quantitative information may be a promising avenue of research for eddy current nondestructive testing.

\section{Acknowledgement and Disclaimer}

Effort sponsored by the Air Force Office of Scientific Research, Air Force Materiel Command, USAF, under grant numbers F49620-93-1-0500, F49620-95-1-0305, and F49620-95-1-0497. The U.S. Government is authorized to reproduce and distribute reprints for Governmental purposes notwithstanding any copyright notation thereon. The views and conclusions contained herein are those of the authors and should not be interpreted as necessarily representing the official policies or endorsements, either expressed or implied, of the Air Force Office of Scientific Research or the U.S. Government. 


\section{References}

[1] M.Y. Antimirov, A.A. Kolyshikin, and R. Vaillancourt, Eddy current nondestructive testing by a perturbation method, Journal of Nondestructive Evaluation, 10 (1991), pp. 31-37.

[2] M.Y. Antimirov, A.A. Kolyshkin, and R. Vaillancourt, Perturbation methods in eddy current testing, in Asymptotic Methods in Mechanics, R. Vaillancourt and A.L. Smirnov editors, Americal Mathematical Society, Providence (1993), pp. 143152.

[3] B.A. Auld, S.R. Jefferies, And J.C. Moulder, Eddy current signal analysis and inversion for semi-elliptical surface cracks, Journal of Nondestructive Evaluation, 7 (1988), pp. 79-94.

[4] J. Blitz, Electrical and Magnetic Methods of Nondestructive Testing, Adam Hilger, Bristol (1991).

[5] A. Calderon, On an inverse boundary value problem, in Seminar on Numerical Analysis and its Applications, W. Meyer and M. Raupp editors, Brazilian Mathematical Society, Rio de Janeiro (1980), pp. 1-7.

[6] G.H. Golub and C.F. Van Loan, Matrix Computations, Johns Hopkins University Press, Baltimore, MD (1983).

[7] P. Lorrain And D.R. Corson, Electromagnetism: Principles and Applications Second Edition, Freeman, New York (1990).

[8] H.L. LiBBy, Introduction to Electromagnetic Nondestructive Test Methods, WileyInterscience, New York (1971).

[9] B. Luong And F. Santosa, Quantitative imaging of corrosion in plates by eddy current methods, in preparation (1996).

[10] L.D. Sabbagh And H.A. Sabbagh, Eddy current modeling and flaw reconstruction, Journal of Nondestructive Evaluation, 7 (1988), pp. 95-110.

[11] M.T. Valley, N.K. Del Grande, and A.S. Kobayashi editors, Nondestructive Inspection of Aging Aircraft SPIE Proceedings, Volume 2001, SPIE, Bellingham (1993). 